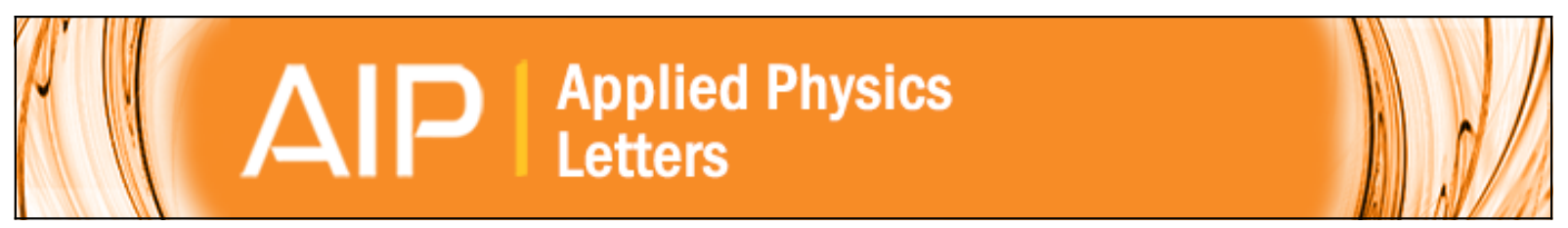

\title{
Quantum-size effects in hafnium-oxide resistive switching
}

Shibing Long, Xiaojuan Lian, Carlo Cagli, Xavier Cartoixà, Riccardo Rurali, Enrique Miranda, David Jiménez, Luca Perniola, Ming Liu, and Jordi Suñé

Citation: Applied Physics Letters 102, 183505 (2013); doi: 10.1063/1.4802265

View online: http://dx.doi.org/10.1063/1.4802265

View Table of Contents: http://scitation.aip.org/content/aip/journal/apl/102/18?ver=pdfcov

Published by the AIP Publishing

\section{AlP Re-register for Table of Content Alerts}

\section{Create a profile.}




\title{
Quantum-size effects in hafnium-oxide resistive switching
}

\author{
Shibing Long, ${ }^{1,2}$ Xiaojuan Lian, ${ }^{1}$ Carlo Cagli, ${ }^{3}$ Xavier Cartoixà, ${ }^{1}$ Riccardo Rurali, ${ }^{4}$ \\ Enrique Miranda, ${ }^{1}$ David Jiménez, ${ }^{1}$ Luca Perniola, ${ }^{3}$ Ming Liu, ${ }^{2}$ and Jordi Suñé $\left.{ }^{1, a}\right)$ \\ ${ }^{1}$ Departament d'Enginyeria Electrònica, Universitat Autònoma de Barcelona, Bellaterra, Spain \\ ${ }^{2}$ Laboratory of Nanofabrication and Novel Device Integration, Institute of Microelectronics, \\ Chinese Academy of Sciences, Beijing, China \\ ${ }^{3}$ CEA-LETI, Grenoble, France \\ ${ }^{4}$ Institut de Ciència de Materials de Barcelona (ICMAB-CSIC), Bellaterra, Spain
}

(Received 25 January 2013; accepted 5 April 2013; published online 7 May 2013)

\begin{abstract}
Discrete changes of conductance of the order of $\mathrm{G}_{0}=2 \mathrm{e}^{2} / \mathrm{h}$ reported during the unipolar reset transitions of $\mathrm{Pt} / \mathrm{HfO}_{2} / \mathrm{Pt}$ structures are interpreted as the signature of atomic-size variations of the conducting filament $(\mathrm{CF})$ nanostructure. Our results suggest that the reset occurs in two phases: a progressive narrowing of the $\mathrm{CF}$ to the limit of a quantum wire $(\mathrm{QW})$ followed by the opening of a spatial gap that exponentially reduces the $\mathrm{CF}$ transmission. First principles calculations show that oxygen vacancy paths in $\mathrm{HfO}_{2}$ with single- to few-atom diameters behave as QWs and are capable of carrying current with $\mathrm{G}_{0}$ conductance. (C) 2013 AIP Publishing LLC. [http://dx.doi.org/10.1063/1.4802265]
\end{abstract}

Simple metal-insulator-metal (MIM) devices show memory properties related to reversible chemical and structural changes that translate into a non-volatile modification of the electrical resistance. ${ }^{1}$ An important feature of these resistive switching (RS) phenomena is the combined implication of ions and electrons. ${ }^{2}$ Electrons are responsible for the conduction, and ions modify the internal state of the device, thus introducing the memory effects. In the case of interest to this Letter, RS is related to the formation and partial destruction of a nanoscale conducting filament (CF). The nature of the $\mathrm{CF}$ is different in different material systems, ${ }^{3,4}$ and so are the conduction and switching properties. ${ }^{1,5-7}$ However, all these systems share some important features, involving the motion of ions and redox reactions, which form and destroy the $\mathrm{CF}$ during the set and reset transitions, respectively. Understanding the physics of the RS phenomena is of great importance to control the performance, variability, and reliability of these devices and to foster their real application as non-volatile memories.

In this Letter, we focus on the reset transition in $\mathrm{Pt} / \mathrm{HfO}_{2} /$ $\mathrm{Pt}$ devices operated under unipolar switching conditions, i.e., the same voltage polarity is used for set and reset. The considered structures are $2.5 \mu \mathrm{m}^{2}$ MIM capacitors fabricated in a mesa structure on top of a tungsten plug. The insulator is a 10-nm-thick $\mathrm{HfO}_{2}$ layer deposited by atomic layer deposition (ALD) at $350{ }^{\circ} \mathrm{C}$ on top of the Pt bottom electrode (BE), followed by Pt top electrode (TE) deposition and patterning. BE and TE were deposited by physical vapor deposition (PVD). Samples with other metal electrodes including Ti and TiN were also considered in the measurements of the temperature dependence of the CF resistance. These RS devices require a preliminary electrical stress usually known as electroforming to activate the switching. In the case of CF-based RS, electroforming is very similar to a soft dielectric breakdown event and consists in the generation of at least one CF. After

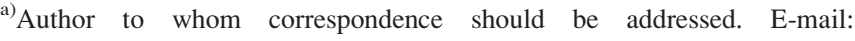
jordi.sune@uab.cat. Telephone: +34-935813527.Fax:+34-935812600
}

electroforming, long lasting repetitive set/reset cycling experiments (1250 consecutive cycles) were performed using a voltage ramp both for set and reset. During the set transition, a compliance current with $1 \mathrm{~mA}$ was applied to avoid the hard breakdown of the $\mathrm{HfO}_{2}$ layer. In the HighResistance-State (HRS), the resistance distribution is quite wide (spanning from $\sim 10^{4} \Omega$ to more than $10^{7} \Omega$ ); while in the Low-Resistance-State (LRS), the resistance is around $10^{2} \Omega$, with much smaller variation. Fig. 1 reports some examples of the current-voltage characteristics measured during typical reset cycles. All these curves show a rather abrupt current drop (particularly when the LRS conductance is high) followed by a progressive current reduction. These curves finally show abrupt jumps between discrete current levels with conductance of the order of few times the quantum of conductance, $\mathrm{G}_{0}=2 \mathrm{e}^{2} / \mathrm{h}$. These results are very similar to those obtained for atomic-sized conductors using different techniques, such as mechanically controllable break junctions or different experiments based on scanning tunneling microscopy. ${ }^{8,9}$

These similarities suggest that the current levels shown in Fig. 1 are related to conductance quantization or to structural variations of the $\mathrm{CF}$ constriction involving the motion of one or very few atoms. On the other hand, all the reset traces show a final drop to zero in the linear current scale of Fig. 1(b), which appears as a change of orders of magnitude in the log scale of Fig. 1(a). Before the final current drop, the $\mathrm{CF}$ behaves as a quantum wire $(\mathrm{QW})$ with at least one conducting channel that contributes with $\sim \mathrm{G}_{0}$ to the $\mathrm{CF}$ conductance. After the final reset event, the conduction is nonlinear because a spatial gap has been opened in the CF and it might be related to thermally assisted tunneling or hopping.

A way to further reveal preferred atomic-scale configurations and/or quantization of the conductance is to study the statistics of conductance readings. Fig. 1(c) shows the histogram of the CF conductance at the point of the final transition to the HRS. At this point, the conductance range is limited to few times $G_{o}$ (two orders of magnitude smaller 

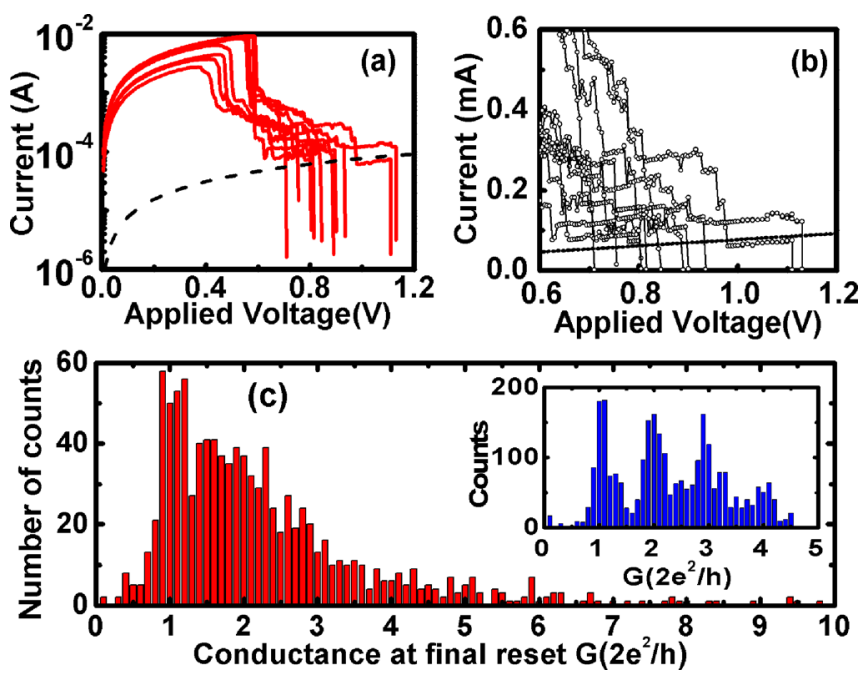

FIG. 1. (a) Current-voltage traces measured during the application of reset voltage ramps. The dashed line corresponds to a linear I(V) with conductance equal to $\mathrm{G}_{0}=2 \mathrm{e}^{2} / \mathrm{h}$. (b) Detail of the current-voltage evolution during the last phase of the reset transients. (c) Histogram of conductance at the final reset point; the inset shows the histogram of conductance readings during 100 successive conductance-time traces.

than in the initial LRS), a clear peak is found at $G \sim G_{0}$ and the probability of higher values of $\mathrm{CF}$ conductance decreases exponentially. This emphasizes that $\mathrm{G}_{0}$ is a natural physicsbased boundary between the LRS and the HRS, and supports the idea that there is an intermediate reset state in which the $\mathrm{CF}$ is a nanoscale filament with properties similar to a $\mathrm{QW}$. However, the existence of preferred CF atomic-scale configurations or of conductance quantization is not revealed by any clear peak structure in Fig. 1(c). Assuming that this might be due to averaging effects related to the drift of the CF shape along the cycling experiment, we have considered a subset of only 100 successive reset cycles. Both the direct observation of the conductance-voltage traces and the conductance histogram (see inset of Fig. 1(c)) clearly reveal the existence of well-defined atomic-size configurations of the CF. The peaks at roughly integer multiples of $G_{0}$ can either be due to the $\mathrm{CF}$ behaving as a $\mathrm{QW}$ or to a nanoscale $\mathrm{CF}$ cross section corresponding to few atomic-size conducting defects.

The change of transport regime when the CF conductance is of the order of $G_{0}$ is confirmed by the temperature dependence of the CF conduction as a function of its conductance. Assuming an Arrhenius model for the temperature dependence, $\mathrm{I}=\mathrm{I}_{\mathrm{O}} \exp \left(-\mathrm{E}_{\mathrm{ACT}} / \mathrm{K}_{\mathrm{B}} \mathrm{T}\right)$, the activation energy $\mathrm{E}_{\mathrm{ACT}}$ measured at low-voltage is reported in Fig. 2(a) to show that the CF conduction is essentially temperature independent when its conductance is above $\mathrm{G}_{0}$ (slightly negative values of $\mathrm{E}_{\mathrm{ACT}}$ account for the metallic-like behavior) while $E_{A C T}$ becomes positive for $G<G_{0}$, a signature of temperature-assisted barrier-limited transport.

The idea that the CF behaves as a QW with two different transport regimes was previously assumed in the quantum point contact model of CF conduction, ${ }^{10,11}$ an extension of a model initially proposed for the post-breakdown conduction through thin gate oxides. ${ }^{12}$ This model was shown to reproduce the experimental current-voltage characteristics in different materials and both in the LRS and the HRS, nicely
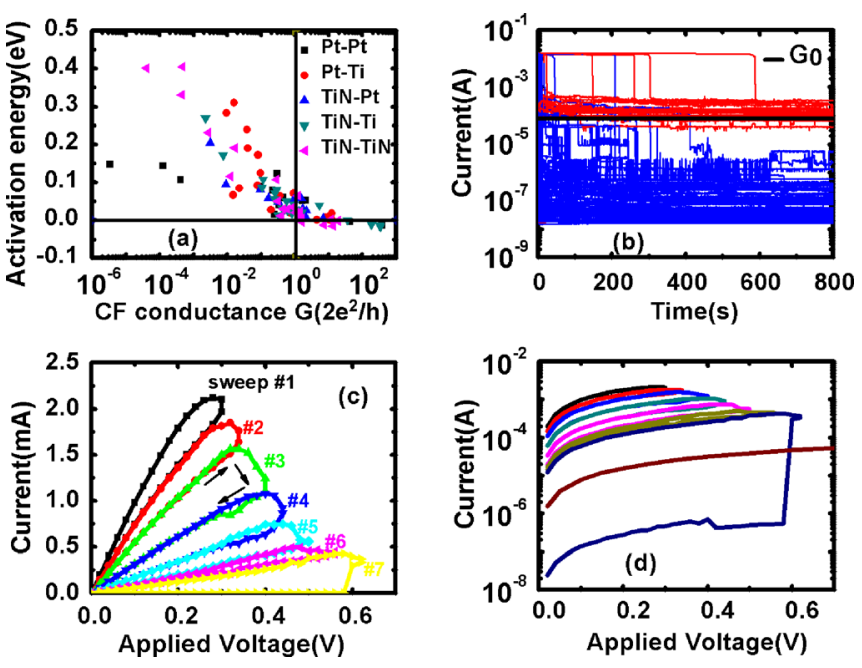

FIG. 2. (a) Activation energy of the low-voltage CF current as a function of the $\mathrm{CF}$ conductance. The vertical line emphasizes the change of transport regime for $\mathrm{CF}$ conductance of the order of $\mathrm{G}_{0}$. (b) Evolution of the CF current during a constant-voltage reset experiment. (c) Control of the transition from the LRS to the intermediate reset state by successive voltage ramps with increasing maximum voltage. From an initial conductance of $125 \mathrm{G}_{0}$, the first six sweeps reduce the $C F$ conductance to $95 G_{0}, 70 G_{0}, 40 G_{0}, 22 G_{0}$, $13 \mathrm{G}_{0}$, and $8 \mathrm{G}_{0}$, respectively. The seventh sweep disrupts the $\mathrm{CF}$ and opens a gap, thus decreasing its conductance by several orders of magnitude, as shown in (d), which depicts the same results in a logarithmic current scale together with a reference line $\left(I=\mathrm{G}_{0} \mathrm{~V}\right)$.

tracking the change from linear to non-linear transport. However, direct evidence of quantum conductance effects has not been reported until very recently and only in systems in which active metal cations form a $\mathrm{CF}$ through a solid electrolyte. ${ }^{13-15}$ The results presented in this Letter suggest that these quantum-size effects also appear in systems in which oxygen vacancies are considered to form the CFs.

The existence of two well-defined transport regimes is also evidenced by constant-voltage stress (CVS) reset experiments. Fig. 2(b) shows that after some time under CVS conditions, a reset transition occurs from the LRS to a lower conductance state. At the end of the stress experiment, the CF conductance is either above (red lines) or orders of magnitude below (blue lines) the quantum of conductance. This indicates that either a narrow $\mathrm{CF}$ remains and supports one or few quantum modes or there is spatial gap in the CF that strongly suppresses the conduction. In other words, these RS structures show three well defined states: (1) the LRS, corresponding to a wide CF with classical metallic properties; (2) a partial reset state in which the CF behaves as a QW and which can be as narrow as a single-defect conducting path; and (3) the HRS, in which a physical gap has been opened in the $\mathrm{CF}$, so that the conduction is non-linear and strongly temperature dependent. The transition from the LRS to the intermediate QW state can be controlled by applying successive voltage ramps with increasing maximum voltage, ${ }^{16}$ as shown in Fig. 2(c). In this particular experiment, the CF conductance is reduced from the initial value of $\sim 125 \mathrm{G}_{0}$ to a conductance of $\sim 8 \mathrm{G}_{0}$ with six successive voltage sweeps. The seventh sweep caused a disconnection of the CF (a spatial gap was opened), and the CF conductance was reduced by orders of magnitude, as shown in Fig. 2(d). Although this technique allows a certain control of the reduction of the $\mathrm{CF}$ 


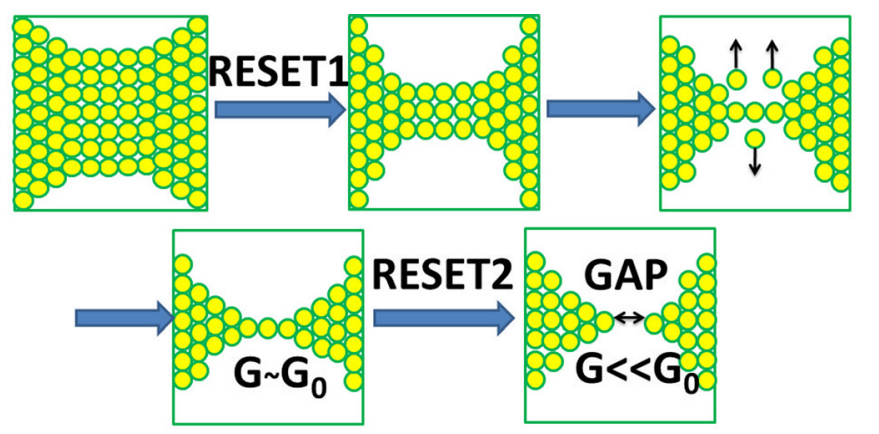

FIG. 3. Schematic representation of the evolution of the CF structure during the reset transient.

diameter when $G>G_{0}$, the resistance of the final HRS is difficult to control because the $\mathrm{CF}$ conductance depends exponentially on the gap length.

A qualitative explanation of the previous results is given in the schematic diagram of Fig. 3. In the LRS, a large number of conducting defects (likely oxygen vacancies) form a wide $\mathrm{CF}$ with metallic conducting properties. During the application of the reset voltage ramp, a critical temperature is reached and induces the first abrupt reset event (RESET 1 ), which in most of the cases causes a significant narrowing of the CF. After this abrupt event, the CF conductance progressively evolves until the $\mathrm{CF}$ is only composed by one or few single defect paths. Then, the final transition to the HRS occurs when a gap is opened in the CF (RESET 2). In this figure, we have arbitrarily assumed that the CF becomes narrower in the center of the insulator film. However, asymmetric $\mathrm{CF}$ shapes are also possible and this should not significantly alter the results provided that the $\mathrm{CF}$ has an atomic-scale size at its most constrictive section.

Oxygen vacancies have been suggested to play a significant role in the RS phenomenon in transition metal oxides with non-active metal electrodes. The results of this Letter suggest that, before the transition to the HRS, the CF behaves as a nanoscale filament with one or few defects in its most constrictive section (or as a bundle of single-defect wide filaments). Therefore, our next step was exploring whether oxygen vacancy paths can explain this type of behavior. To address this, we have carried out first-principles calculations in order to obtain the electronic structure of oxygen vacancy filaments in a crystalline $\mathrm{HfO}_{2}$ host. These calculations are made within the density-functional theory (DFT), as implemented in the SIESTA package. ${ }^{17,18}$ In order to minimize the coupling between the filament instances, which we take to be along the $\mathbf{c}$ axis for the crystalline material (see Ref. 19 for the definition of the axes), we use a $3 \times 3$ supercell of the monoclinic unit cell- the stable phase up to $1720^{\circ} \mathrm{C}$ - in the ab directions, sampling them in the Brillouin zone with a grid of $2 \times 2$ of $\mathbf{k}$-points within the Monkhorst-Pack algorithm. ${ }^{20}$ All the structures discussed have been relaxed until all the forces on the atoms were lower than $0.04 \mathrm{eV} / \AA$. The ballistic conductance is calculated from first-principles within Landauer theory. ${ }^{21}$ Open boundary conditions are accounted for through the left (right) self-energy. The zero-bias transmission $\mathrm{T}(\mathrm{E})$ is calculated using non-equilibrium Green's functions, and the conductance is then calculated through the Landauer formula as $\mathrm{G}=\mathrm{T}(\mathrm{E}) \mathrm{G}_{0}$.
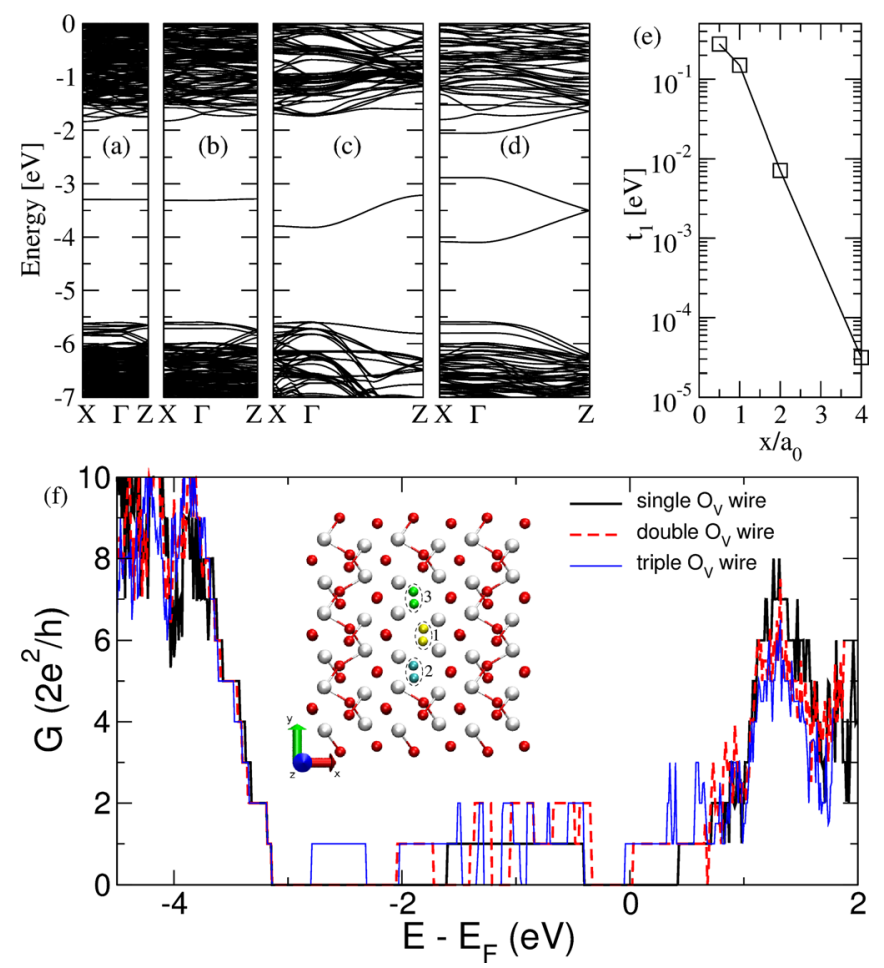

FIG. 4. Band structure for crystalline $\mathrm{m}-\mathrm{HfO}_{2}$ with $\mathrm{O}$ vacancies separated by (a) $4 a_{0}$, (b) $2 a_{0}$, (c) $a_{0}$ and (d) $a_{0} / 2$. (e) Hopping parameter as a function of vacancy-vacancy separation. (f) Conductance as a function of energy corresponding to a $\mathrm{HfO}_{2}$ matrix where one, two, or three $\mathrm{O}$ atom rows are removed. The rows subsequently removed are shown in the instate (marked as " 1 ," "2," and " 3 "), where red and white spheres correspond to O and Hf atoms, respectively.

The removal of a single $\mathrm{O}$ atom in a monoclinic- $\mathrm{HfO}_{2}$ $\left(\mathrm{m}-\mathrm{HfO}_{2}\right)$ matrix introduces a filled impurity state in the gap, similarly to $\mathrm{TiO}_{2},{ }^{22}$ but farther from the band edges [see Fig. 4(a)]. The spatial extent of the impurity wavefunction determines the state overlap between two neighboring vacancies and thus relates to the transition from hopping to band transport. Figures 4(a)-4(d) show the band structure of $\mathrm{m}-\mathrm{HfO}_{2}$ with a chain of oxygen vacancies that are increasingly close together. It can be seen that, as the overlap between the impurity wavefunctions increases, the impurity band width increases as well. This can be well described with a single band, second-neighbor one-dimensional (1D) tight-binding Hamiltonian, which is well known to have the dispersion relation

$$
E\left(k_{x}\right)=E_{0}-2 t_{1} \cos \left(k_{x} \frac{a_{o}}{2}\right)-2 t_{2} \cos \left(k_{x} a_{o}\right),
$$

where $E_{0}$ is the isolated impurity energy, $t_{1}$ and $t_{2}$ are the first and second neighbor hopping parameters, closely related to the amount of overlap between the wavefunctions of neighboring impurities, and $\mathrm{a}_{0}$ is the length of the c-axis vector for the $\mathrm{m}-\mathrm{HfO}_{2}$ primitive cell $(5.296 \AA)$. Fig. 4(e) shows the behavior of $t_{1}$ as the vacancy-vacancy distance varies, showing the expected exponential decay from which a localization parameter $\chi=0.36 \mathrm{a}_{0}$ can be extracted. From the Mott criterion for metal-to-insulator transitions, ${ }^{23}$ we can find that the critical vacancy concentration is $\sim 1.5 \times 10^{21} \mathrm{~cm}^{-3}$, i.e., a local composition $\mathrm{HfO}_{2-\mathrm{x}}$ with $\mathrm{x}=0.05$ will be enough to enable the band transport mechanism. 
Fig. 4(f) shows how atomic-sized changes in the CF diameter bring out significant increases in the conductance. Specifically, we show the intrinsic conductance (i.e., without the effects of the metallic electrodes) for increasingly wider CFs. We remove one to three oxygen columns (see inset), observing that conductance increases stepwise for certain energy ranges, with each transmitting channel contributing a quantum of conductance $\mathrm{G}_{0}$. This supports our interpretation that the observed quantization of the experimental data arises from few/single atom changes in the atomic structure of the $\mathrm{CF}$. These results have to be taken qualitatively, as the model considered is simplified for a number of reasons (no metal contacts, zero bias conditions). However, we believe that they are able to capture the essential physics, namely that atomic size modifications of the structure of the $\mathrm{CF}$ will give rise to changes in the quantized conductance that are qualitatively consistent with the experimental observations.

In conclusion, atomic size effects have been reported during the reset of $\mathrm{CFs}$ in $\mathrm{Pt} / \mathrm{HfO}_{2} / \mathrm{Pt}$ structures. The existence of a rather stable $\mathrm{CF}$ intermediate state between the LRS and the HRS has been demonstrated. In this state, the CF behaves as a QW with conductance of the order of the quantum of conductance and the existence of preferred atomic-scale configurations has been revealed by conductance histograms. Our results indicate that the reset begins by a progressive narrowing of the $\mathrm{CF}$ towards the limit of one single-atom chain with conductance of about $\mathrm{G}_{0}$. Then, a spatial gap is opened and the CF switches to the HRS, with its conductance dropping orders of magnitude. Thus, the quantum of conductance is a natural boundary between the LRS and HRS. The temperature dependence of the CF transport properties also confirm that the transition from metallic to temperature activated transport occurs for a $\mathrm{CF}$ conductance of the order of $G_{0}$. The study of the transport properties of oxygen vacancy paths using ab-initio methods shows that they are able to introduce band transport (as opposed to hopping transport) and support multiple transport channels with conductance $G_{o}$ related to increasing path width. Furthermore, increasing the distance between vacancies has been shown to exponentially decrease the hopping parameter. This indicates that opening a gap in these vacancy paths would decrease the CF conductance exponentially, as it is observed in the experimental transition to the HRS.

This work was funded by Spanish Ministry of Science and Technology under Contract TEC2012-32305 (partially supported by the EU under the FEDER Program), FIS200912721-C04-03, and CSD2007-00041, the DURSI of the Generalitat de Catalunya under Contract 2009SGR783, the Ministry of Science and Technology of China under Grant Nos. 2010CB934200, 2011CBA00602, 2009CB925003, 2011CB921804, 2011AA010401, and 2011AA010402, and the National Natural Science Foundation of China under Grant Nos. 61221004, 60825403, 61274091, 50972160, 61106119, and 61106082. J. Suñé also thanks the funding support of the ICREA ACADEMIA Award. Devices and data have been obtained in the frame of internal CEA-LETI research programs.

${ }^{1}$ R. Waser, R. Dittmann, G. Staikov, and K. Szot, Adv. Mater. 21, 2632 (2009).

${ }^{2}$ M. Di Ventra and Y. V. Pershin, Mater. Today 14, 584 (2011).

${ }^{3}$ I. K. Yoo, B. S. Kang, S. E. Ahn, C. B. Lee, M. J. Lee, G. S. Park, and X. S. Li, IEEE Trans. Nanotechnol. 9, 131 (2010).

${ }^{4}$ D. H. Kwon, K. M. Kim, J. H. Jang, J. M. Jeon, M. H. Lee, G. H. Kim, X.-S. Li, G.-S. Park, B. Lee, S. Han, M. Kim, and C. S. Hwang, Nature Nanotechnology 5, 148 (2011).

${ }^{5}$ I. Valov, R. Waser, J. R. Jameson, and M. N. Kozicki, Nanotechnology 22, 254003 (2011).

${ }^{6}$ K. Szot, M. Rogala, W. Speier, Z. Klusek, A. Besmehn, and R. Waser, Nanotechnology 22, 254001 (2011).

${ }^{7}$ D. Ielmini, R. Bruchhaus, and R. Waser, Phase Trans. 84, 570 (2011).

${ }^{8}$ N. Agrait, A. Levy-Yeyati, J. M. van Ruitenbeek, Phys. Rep. 377, 81 (2003).

${ }^{9}$ T. Schmidt, R. Martel, R. L. Sandstrom, and P. Avouris, Appl. Phys. Lett. 73, 2173 (1998).

${ }^{10}$ E. Miranda, C. Walczyk, C. Wenger, and T. Schroeder, IEEE Electron Device Lett. 31, 609 (2010).

${ }^{11}$ R. Degraeve, Ph. Roussel, L. Goux, D. Wouters, J. Kittl, L. Altimine, M. Jurczak, and G. Groseneken, Tech. Dig.-Int. Electron Devices Meet. 2010, 632.

${ }^{12}$ J. Suñé, E. Miranda, M. Nafria, and X. Aymerich, Tech. Dig.-Int. Electron Devices Meet. 1998, 191.

${ }^{13}$ J. R. Jameson, N. Gilbert, F. Koushan, J. Saenz, J. Wang, S. Hollmer, M. Kozicki, and N. Derhacobian, IEEE Electron Device Lett. 33, 257 (2012).

${ }^{14}$ X. Zhu, W. Su, Y. Liu, B. Hu, L. Pan, W. Lu, J. Zhang, and R.-W. Li, Adv. Mater. 24, 3941 (2012).

${ }^{15}$ S. Tappertzhofen, I. Valov, and R. Waser, Nanotechnology 23, 145703 (2012).

${ }^{16}$ D. Ielmini, F. Nardi, and C. Cagli, Nanotechnology 22, 254022 (2011).

${ }^{17}$ J. M. Soler, E. Artacho, J. D. Gale, A. García, J. Junquera, P. Ordejón, and D. Sánchez-Portal, J. Phys.: Condens. Mater. 14, 2745 (2002).

${ }^{18}$ J. P. Perdew, K. Burke, and M. Ernzerhof, Phys. Rev. Lett. 77, 3865 (1996).

${ }^{19}$ X. Zhao and D. Vanderbilt, Phys. Rev. B 65, 233106 (2002).

${ }^{20}$ H. J. Monkhorst and J. D. Pack, Phys. Rev. B 13, 5188 (1976).

${ }^{21}$ M. Brandbyge, J.-L. Mozos, P. Ordejón, J. Taylor, and K. Stokbro, Phys. Rev. B 65, 165401 (2002).

${ }^{22}$ S.-G. Park, B. Magyari-Köpe, and Y. Nishi, Phys. Rev. B 82, 115109 (2010).

${ }^{23}$ N. F. Mott, Metal-Insulator Transitions, 2nd ed. (Taylor \& Francis, London, 1990). 